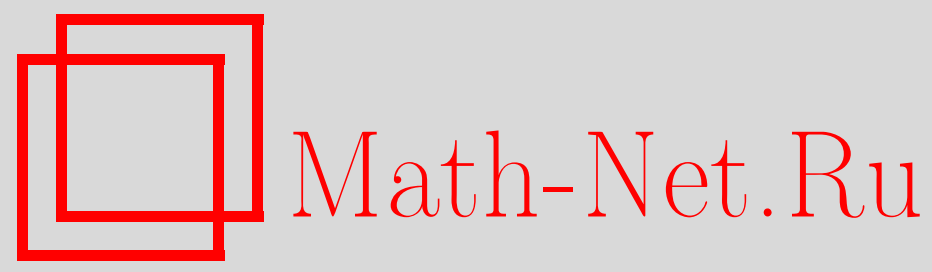

А. С. Кондратьев, Нормализаторы силовских 2-подгрупп в конечных простых группах, Матем. заметки, 2005, том 78, выпуск 3, 368-376

DOI: https://doi.org/10.4213/mzm2593

Использование Общероссийского математического портала Math-Net.Ru подразумевает, что вы прочитали и согласны с пользовательским соглашением http://www . mathnet.ru/rus/agreement

Параметры загрузки:

IP : 54.224 .135 .184

26 апреля 2023 г., 05:12:48 


\title{
НОРМАЛИЗАТОРЫ СИЛОВСКИХ 2-ПОДГРУПП В КОНЕЧНЫХ ПРОСТЫХ ГРУППАХ
}

\section{А. С. Кондратьев}

\begin{abstract}
Завершается описание нормализаторов силовских 2-подгрупп в конечных простых группах.

Библиография: 13 названий.
\end{abstract}

1. Введение. Хорошо известно большое влияние свойств нормализаторов 2-подгрупп на строение конечной неразрешимой групшы. Особьй интерес вызывают нормализаторы силовских 2-подгрупп в группах, близких к конечным простьг группам. Еще $\Phi$. Холл (см. [1, лемма 4]) доказал самонормализуемость силовских 2-подгрупп в симметрических группах $S_{n}$. Нормализаторы силовских 2-подгрупп в конечных простых группах лиева типа над полем характеристики 2 хорошо известны, это подгруппы Бореля. Р. Картер и П. Фонг [1] описали нормализаторы силовских 2-подгрупп в расширенных классических группах над конечными полями нечетной характеристики. Недавно автор и В. Д. Мазуров [2] попутно с описанием максимальных 2-сигнализаторов во всех конечньх простых групах получили описание в них централизаторов силовских 2-подгрупп, а также выяснили строение нормализаторов силовских 2-подгрупп в конечных простых группах исключительного лиева типа над полем нечетной характеристики. Естественно возникающий вопрос о строении нормализаторов силовских 2-подгрупп в остальных конечных простых группах оставался до конца не решенным (см., например, $[3,(6.3)])$. В данной работе завершается решение этого вопроса. Доказаны следующие три теоремы.

Tеорема 1. Пусть $G$ - одна из конечных классических групп $G L_{m}(q), G U_{m}(q)$, $S p_{2 m}(q), G O_{2 m+1}(q), G O_{2 m}^{ \pm}(q)$, где $m \geqslant 1$ u $q$ - степень нечетного простого числа р. Пусть $T$ - силовская 2-подгруппа в $G$ и $T^{0}=T \cap O^{p^{\prime}}(G)$. Тогда $N_{G}\left(T^{0}\right)=$ $T C_{G}(T)$, за исключением следующих случаев:

(a) $G=G L_{2}(q)$ илu $G U_{2}(q), q \equiv \pm 3(\bmod 8)$ u $N_{G}\left(T^{0}\right) / Z(G) \cong S_{4}$;

(б) $G=S p_{2 m}(q), q \equiv \pm 3(\bmod 8)$ и факторгруппа $N_{G}\left(T^{0}\right) / T^{0}$ изоморфна әлементарной абелевой 3-группе порядка $3^{t}$, әде число $t$ находится из 2-адического разложения $m=2^{s_{1}}+\cdots+2^{s_{t}}, s_{1}>\cdots>s_{t} \geqslant 0$;

(в) $G=G O_{3}(q) \cong 2 \times P G L_{2}(q), q \equiv \pm 3(\bmod 8) u N_{G}\left(T^{0}\right) / Z(G) \cong S_{4}$;

Работа выполнена при поддержке Российского фонда фундаментальных исследований, гранты № 04-01-00463 и № 04-01-81001. 
(г) $G=G O_{2}^{ \pm}(q) \cong D_{2(q \mp 1)}, q \mp 1$ не является степенью 2 и $N_{G}\left(T^{0}\right)=G$;

(д) $G=G O_{4}^{+}(q) \cong\left(S L_{2}(q) \circ S L_{2}(q)\right) \cdot 2^{2}, q \equiv \pm 3(\bmod 8) u N_{G}\left(T^{0}\right) \cong G O_{4}^{+}(3)$;

(e) $G=G O_{5}(q) \cong 2 \times\left(P S p_{4}(q) .2\right), q \equiv \pm 3(\bmod 8) u N_{G}\left(T^{0}\right) / Z(G) \cong G O_{4}^{+}(3)$.

Из теоремы 1 следует вышеупомянутый результат Картера и Фонга. Доказательство теоремы 1 основано на результатах М. Ашбахера [3], [4] о фундаментальных подгруппах в конечных группах лиева типа над полем нечетной характеристики, а также на результатах о стабилизаторах ортогональных разложений в конечных классических группах (см. [5], [6]).

По аналогии с доказательством теоремы 1 доказывается

Tеорема 2. Пусть $T$ - силовская 2-подгруппа в $S_{n} u T^{0}=T \cap A_{n}$. Тогда $N_{S_{n}}\left(T^{0}\right)=T$, за исключением следуюших случаев:

(a) $n=3 u N_{S_{3}}\left(T^{0}\right)=S_{3}$;

(б) $n=4$ или 5 u $N_{S_{n}}\left(T^{0}\right) \cong S_{4}$.

Из теоремы 2 следует вышеупомянутьй результат Холла.

С использованием [7] и теорем 1 и 2 легко доказьвается

Tеорема 3. Пусть $G$ - одна из конечных простых спорадических групп, $A=$ Aut $(G), T$ - силовская 2-подгруппа в $A$ и $T^{0}=T \cap G$. Тогда $N_{A}\left(T^{0}\right)=T$, за исключением следующих случаев:

(a) $G=A=J_{1}$ u $N_{A}\left(T^{0}\right) \cong 2^{3} .7 .3<\operatorname{Hol}\left(2^{3}\right)$;

(б) $G=J_{2}, J_{3}$, Suz или $H N,|A: G|=2 u N_{A}\left(T^{0}\right) / T^{0} \cong S_{3}$.

Из теорем $1-3$ и $[2$, теоремы 6 и 7$]$ непосредственно вытекает

СледствиЕ. Пусть $G$ - конечная простая неабелева группа и $S$ - силовская 2-подгруппа в $G$. Тогда $N_{G}(S)=S$, за исключением следующих случаев:

(a) $G$ - группа лиева типа над полем характеристики 2 и $N_{G}(S)$ - подгруппа Бореля в $G$, отличная от $S$;

(б) $G \cong L_{2}(q), 3<q \equiv \pm 3(\bmod 8) u N_{G}(S) \cong A_{4}$;

(в) $G \cong L_{m}^{ \pm}(q), m \geqslant 3$, q нечетно и $S \neq N_{G}(S)=S \times C_{1} \times \cdots \times C_{t-1}$, где число $t \geqslant 2$ находится из 2-адического разложения $m=2^{s_{1}}+\cdots+2^{s_{t}}$, $s_{1}>\cdots>s_{t} \geqslant 0$, a $C_{1}, \ldots, C_{t-2}, C_{t-1}$ - чиклические группы порядков $(q \mp 1)_{2^{\prime}}, \ldots,(q \mp 1)_{2^{\prime}},(q \mp 1)_{2^{\prime}} /(q \mp 1, m)_{2^{\prime}}$ соответственно (здесь $L_{m}^{+}(q) u$ $L_{m}^{-}(q)$ обозначают соответственно группы $L_{m}(q)$ и $\left.U_{m}(q)\right)$;

(г) $G \cong P S p_{2 m}(q), m \geqslant 2, q \equiv \pm 3(\bmod 8)$ и факторгруппа $N_{G}(S) / S$ изоморфна әлементарной абелевой 3-группе порядка $3^{t}$, где число $t$ находится из 2-адического разложсения $m=2^{s_{1}}+\cdots+2^{s_{t}}, s_{1}>\cdots>s_{t} \geqslant 0$;

(д) $G \cong E_{6}^{ \pm}(q)$, q нечетно и $N_{G}(S)=S \times C$, где $C$ - неединичная ииклическая группа порядка $(q \mp 1)_{2^{\prime}} /(q \mp 1,3)$ (здесь $E_{6}^{+}(q)$ и $E_{6}^{-}(q)$ обозначают coответственно группы $\left.E_{6}(q) u^{2} E_{6}(q)\right)$;

(e) $G \cong{ }^{2} G_{2}(q)$ или $J_{1}$ u $N_{G}(S) \cong 2^{3} .7 .3<\operatorname{Hol}\left(2^{3}\right)$;

(ж) $G \cong J_{2}, J_{3}$, Suz или $H N$ u $\left|N_{G}(S): S\right|=3$.

Заметим, что теорему 7 из [2] для случая классических групп можно получить из доказательства теоремы 1. 
Наши обозначения и терминология в основном стандартны; их можно найти в [7]-[9]. Если группа $G$ действует на множестве $X$ и $Y \subset X$, то через $G_{Y}$ и $G_{(Y)}$ обозначим соответственно глобальньй и поточечный стабилизатор подмножества $Y$ в $G$, через $C_{X}(G)-$ множество всех фиксируемых $G$ точек из $X$, а через $G^{X}$ - группу подстановок, индуцируемую $G$ на $X$, т.е. $G^{X} \cong G / G_{(X)}$. Если $A$ и $B$ - группы и $n$ - натуральное число, то через $A . B$ (соответственно $A: B$ ) обозначается расширение (соответственно расшепляемое расширение) групшы $A$ посредством групшы $B$, через $A \circ B$ - некоторое их нетривиальное центральное произведение, через $A^{n}$ - прямое произведение $n$ изоморфных $A$ групп. Если $n$ - натуральное число и $r$ - простое число, то $n$ и $r^{n}$ будут обозначать также соответственно циклическую групу порядка $n$ и элементарную абелеву $r$-групу порядка $r^{n}$. Если $A$ - конечная группа и $r$ - простое число, то через $O^{r^{\prime}}(A)$ обозначается подгруппа, порожденная всеми $r$-элементами группы $A$.

2. Доказательство теоремы 1. Пусть $q=p^{f}$ обозначает натуральную степень нечетного простого числа $p$ и $I=G$ - одна из классических групп $G L_{n}(q), G U_{n}(q)$, $S p_{n}(q), G O_{n}^{\delta}(q)$, где $\delta$-пустой символ при нечетном $n$ и $\delta= \pm$ при четном $n$. Пусть $V-$ ассоциированное с $I$ векторное пространство размерности $n$ надполем порядка $q$ или $q^{2}$ в случае $I=G U_{n}(q)$, снабженное (при $I \neq G L_{n}(q)$ ) соответствующей формой, такое, что $I$ можно отождествить при выборе подходящей базы в $V$ с соответствующей группой $I(V) \in\left\{G L(V), G U(V), S p(V), G O^{\delta}(V)\right\}$, совпадающей с группой всех невырожденных линейных преобразований пространства $V$, сохраняющих (при $\left.I \neq G L_{n}(q)\right)$ заданную форму. Групша $I$ называется (полной) әруппой изометрий пространства $V$. Положим также

$$
S=S(V)=I(V) \cap S L(V) \in\left\{S L(V), S U(V), S p(V), S O^{\delta}(V)\right\}
$$

и

$$
\Omega=\Omega(V)=O^{p^{\prime}}(S(V))
$$

Чepe3

$$
\begin{aligned}
& P I(V) \in\left\{P G L(V), P G U(V), P S p(V), P G O^{\delta}(V)\right\} \\
& P S(V) \in\left\{P S L(V), P S U(V), P S p(V), P S O^{\delta}(V)\right\}
\end{aligned}
$$

и

$$
P \Omega(V) \in\left\{P S L(V), P S U(V), P S p(V), P \Omega^{\delta}(V)\right\}
$$

обозначим образы групा $I(V), S(V)$ и $\Omega(V)$ соответственно при естественном гомоморфизме грушшы $I$ на факторгрупу $I / Z(I)$. Для краткости обозначим через $G L^{\delta}(V)$, $S L^{\delta}(V)$ и $L^{\delta}(V)$ соответственно групшы $G L(V), S L(V)$ и $P S L(V)$ при $\delta=+$ и $G U(V)$, $S U(V)$ и $P S U(V)$ при $\delta=-$.

Разложение $V=U_{1} \oplus \cdots \oplus U_{m}$ в виде ортогональной (относительно заданной на $V$ формы) суммы (прямой суммы при $I=G L(V))$ подпространств $U_{1}, \ldots, U_{m}$ назьвается ортогональным разложсением.

Если $n=1$, то $I=G L_{1}^{\delta}(q)$ или $G O_{1}(q)$, т.е. $I$ - циклическая группа порядка $q-\delta 1$ или 2 соответственно, откуда $\Omega=1$, и поэтому в этом случае теорема верна.

Если $n=2$, то $I=G L_{2}^{\delta}(q), S p_{2}(q)$ или $G O_{2}^{\delta}(q)$, откуда ввиду [6, предложение 2.9.1] группа $I$ изоморфна $\left((q-\delta 1) \circ S L_{2}(q)\right) .2, S L_{2}(q)$ или $D_{2(q-\delta 1)}$ соответственно, причем 
$P G L_{2}^{\delta}(q) \cong P G L_{2}(q)$, и поэтому справедливость теоремы в этом случае вытекает из хорошо известного описания подгрупп в $P S L_{2}(q)$ (см. [10, глава 12]) и диэдральности силовской 2-подгрупшы в $P G L_{2}(q)$.

Если $I=G O_{3}(q)$, то по [6, предложение 2.9.1] $I=Z(I) \times S O_{3}(q) \cong 2 \times P G L_{2}(q)$, и поэтому в этом случае теорема верна.

Пусть $I=G O_{4}^{+}(q)$. Тогда ввиду [6, предложения 2.5.6, 2.5.8, 2.9.1 и 2.9.4] $I=$ $\left(L_{1} \circ L_{2}\right)\langle s, r\rangle$, где $L_{1} \cong L_{2} \cong S L_{2}(q), \Omega=L_{1} \circ L_{2}, S=\Omega\langle s\rangle,|I: S|=|S: \Omega|=2$, отражение $r$ переставляет подгруппы $L_{1}$ и $L_{2}$, а элемент $s$ индуцирует на $L_{1}$ и $L_{2}$ внешний диагональный автоморфизм. Отсюда видно, что в этом случае теорема верна.

Пусть $I=G O_{4}^{-}(q)$. Тогда ввиду [6, таблища 2.1.С и предложение 2.9.1] $|I: S|=$ $|S: \Omega|=2$ и $\Omega \cong L_{2}\left(q^{2}\right)$. Отсюда видно, что в этом случае теорема верна.

В дальнейшем мы будем предполагать $n$ не меньше $3,4,5$ в случаях $I=G L_{n}^{\delta}(q)$, $S p_{n}(q), G O_{n}^{\delta}(q)$ соответственно. Хорошо известно (см., например, [6, предложения 2.1.3 и 2.9.1]), что тогда $P \Omega$ - неабелева простая группа.

Приведем необходимые нам понятия и результаты Ашбахера [3], [4], связанные с фундаментальными подгруппами групшы $\Omega$, рассматриваемой как группа лиева типа над полем порядка $q$.

Пусть $U$ - длинная корневая подгрупша в $\Omega, U^{-}-$противоположная ей подгруппа и $K=\left\langle U, U^{-}\right\rangle$. Тогда подгруппа $K$ изоморфна $S L_{2}(q)$. Класс сопряженных с $K$ подгрупा в $\Omega$ называется множеством фундаментальны $x$ оодрупn в $\Omega$. Пусть $T$ - фиксированная силовская подгруппа в $I$ и $T^{0}=T \cap \Omega$. Положим

$$
\Delta=\operatorname{Fun}_{\Omega}\left(T^{0}\right)=\left\{K^{x} \mid x \in \Omega, K^{x} \cap T^{0} \in \operatorname{Syl}_{2}\left(K^{x}\right)\right\}
$$

Тогда $\Delta$ - максимальное множество попарно коммутирующих фундаментальных подгрупп из $\Omega$. Из определения множества $\Delta$ непосредственно видно, что $N_{\Omega}\left(T^{0}\right) \leqslant N_{\Omega}(\Delta)$.

Пусть $I=G L^{\delta}(V)$ или $S p(V)$ и $n=2 k+a$ с $0 \leqslant a \leqslant 1$. По [3, (2.1)] существует ортогональное разложение

$$
V=V_{0} \oplus V_{1} \oplus \cdots \oplus V_{k}
$$

такое, что $\operatorname{dim} V_{i}=2$ для $1 \leqslant i \leqslant k, \operatorname{dim} V_{0}=a$ и $\Delta=\left\{K_{i} \mid 1 \leqslant i \leqslant k\right\}$, где $K_{i}=S\left(V_{i}\right)$ и $I\left(V_{i}\right)$ обозначает подгруппу из $I(V)$, действующую точно на $V_{i}$ как ее соответствующая полная групп изометрий и централизующую каждое подпространство $V_{j}$ при $j \neq i$. Кроме того, $N_{\Omega}(\Delta)^{\Delta} \cong S_{k}$. Положим $\mathscr{D}=\left\{V_{0}, V_{1}, \ldots, V_{k}\right\}$ и $W=V_{1} \oplus \cdots \oplus V_{k}$. Так как $\left[S\left(V_{i}\right), V\right]=V_{i}$ и $I\left(V_{i}\right) S=I$ для $1 \leqslant i \leqslant k$, а $V_{0}=\bigcap_{i=1}^{k} C_{V}\left(S\left(V_{i}\right)\right)$, то $N_{I}\left(T^{0}\right) \leqslant N_{I}(\Delta) \leqslant I_{\mathscr{D}}$.

Пусть $I=G L^{\delta}(V)$. Тогда по [6, предложения 4.1.4 и 4.2.9]

$$
\begin{gathered}
N_{I}(\Delta)=I_{\mathscr{D}}=I\left(V_{0}\right) \times\left(\left(G L^{\delta}\left(V_{1}\right) \times \cdots \times G L^{\delta}\left(V_{k}\right)\right): S_{k}\right) \cong(q-\delta 1)^{a} \times\left(G L_{2}^{\delta}(q) \succ S_{k}\right), \\
I^{\mathscr{D}}=S^{\mathscr{D}} \cong S_{k}, \quad I_{\mathscr{D}} / C_{I_{(\mathscr{D})}}(\langle\Delta\rangle) \cong P G L_{2}(q)<S_{k}
\end{gathered}
$$

И

$$
S_{(\mathscr{D})} / C_{S_{(\mathscr{D})}}(\langle\Delta\rangle) \cong L_{2}(q)^{k} \cdot 2^{k-1+a},
$$

причем

$$
N_{S_{(\mathscr{D})}}\left(K_{i}\right) / C_{S_{(\mathscr{D})}}\left(K_{i}\right) \cong P G L_{2}(q), \quad 1 \leqslant i \leqslant k .
$$


Ввиду самонормализуемости силовских 2-подгрупш в групшах $S_{k}$ и $P G L_{2}(q)$ отсюда следует, что $N_{I}\left(T^{0}\right)=T C_{I}(T)$, т.е. теорема в этом случае верна.

Пусть $I=S p(V)$. Тогда $I=S$ и $T=T^{0}$. По [6, предложение 4.2.10]

$$
\begin{aligned}
& N_{I}(\Delta)=I_{\mathscr{D}}=\left(S p\left(V_{1}\right) \times \cdots \times S p\left(V_{k}\right)\right): S_{k} \cong S p_{2}(q)<S_{k} \\
& I^{\mathscr{D}} \cong S_{k} \quad \text { и } \quad I_{\mathscr{D}} / Z(\langle\Delta\rangle) \cong L_{2}(q)<S_{k} .
\end{aligned}
$$

Так как силовские 2 -подгрупшы в $S_{k}$ самонормализуемы, можно считать, что

$$
N_{I}(T) \leqslant\left(N_{K_{1}}\left(T \cap K_{1}\right) \times \cdots \times N_{K_{k}}\left(T \cap K_{k}\right)\right):\left(T \cap S_{k}\right)
$$

Если $q \not \equiv \pm 3(\bmod 8)$, то $N_{K_{i}}\left(T \cap K_{i}\right)=T \cap K_{i}, 1 \leqslant i \leqslant k$, откуда $N_{I}(T)=T$ и теорема верна. Пусть $q \equiv \pm 3(\bmod 8)$ и $k=2^{s_{1}}+\cdots+2^{s_{t}}, s_{1}>\cdots>s_{t} \geqslant 0$. Тогда $N_{K_{i}}\left(T \cap K_{i}\right) \cong S L_{2}(3)$ и $T \cap S_{k} \cong H_{s_{1}} \times \cdots \times H_{s_{t}}$, где $H_{s}$ обозначает последовательное сплетение групшы порядка 2 с самой собой $s$ раз. Пусть $x_{i}-$ элемент порядка 3 из $N_{K_{i}}\left(T \cap K_{i}\right)$. Тогда можно считать, что группа $T \cap S_{k}$ нормализует множество $\left\{x_{i} \mid 1 \leqslant i \leqslant k\right\}$ и имеет на нем орбиты с длинами $2^{s_{1}}, \ldots, 2^{s_{t}}$. В каждой такой орбите группа $T \cap S_{k}$ фиксирует единственный элемент. Отсюда следует утверждение теоремы (случай (б)).

Пусть $I=G O^{\delta}(V)$ и $n=4 k+a$ с $0 \leqslant a \leqslant 4, \delta=+$ при $a=0$ и $\delta=-$ при $a=4$. По $[3,(2.2)]$ существует ортогональное разложение

$$
V=V_{0} \oplus V_{1} \oplus \cdots \oplus V_{k}
$$

такое, что $V_{i}$ имеет размерность 4 и знак + для $1 \leqslant i \leqslant k, \operatorname{dim} V_{0}=a$ и $\Delta=\left\{K_{i}, K_{i}^{*} \mid\right.$ $1 \leqslant i \leqslant k\}$, где $K_{i}$ 。 $K_{i}^{*} \cong \Omega^{+}\left(V_{i}\right)$ и $I\left(V_{i}\right)$ обозначает подгруппу из $I(V)$, действующую точно на $V_{i}$ как полная группа изометрий $G O_{4}^{+}(q)$ и централизующую каждое подпространство $V_{j}$ при $j \neq i$. Кроме того, группа $N_{\Omega}(\Delta)^{\Delta}$ изоморфна $2^{k} . S_{k}$ при $a \neq 0$ и $2^{k-1} . S_{k}$ при $a=0$, причем $N_{\Omega}(\Delta)^{\Delta}$ действует на $\Delta$ импримитивно с системой импримитивности $\Gamma=\left\{\left\{K_{i}, K_{i}^{*}\right\} \mid 1 \leqslant i \leqslant k\right\}$ и индуцирует на $Г$ группу подстановок $S_{k}$.

Положим $\mathscr{D}=\left\{V_{0}, V_{1}, \ldots, V_{k}\right\}$ и $W=V_{1} \oplus \cdots \oplus V_{k}$. Так как $\left[\Omega\left(V_{i}\right), V\right]=V_{i}$ и $I\left(V_{i}\right) \Omega=I$ для $1 \leqslant i \leqslant k$, а $V_{0}=\bigcap_{i=1}^{k} C_{V}\left(\Omega\left(V_{i}\right)\right)$, то $N_{I}\left(T_{0}\right) \leqslant N_{I}(\Delta) \leqslant I_{\mathscr{D}}$.

Пусть сначала $I=G O^{+}(V)$ и $a=0$, т.е. $n=4 k \geqslant 8$. Тогда по [6, предложение 4.2.11]

$$
\begin{gathered}
N_{I}(\Delta)=I_{\mathscr{D}}=\left(G O^{+}\left(V_{1}\right) \times \cdots \times G O^{+}\left(V_{k}\right)\right): S_{k} \cong G O_{4}^{+}(q)<S_{k}, \\
I^{\mathscr{D}}=\Omega^{\mathscr{D}} \cong S_{k}, \quad I_{(\mathscr{D})} \Omega=I, \quad I_{(\mathscr{D})} / \Omega_{(\mathscr{D})} \cong I / \Omega \cong 2^{2}, \\
I_{(\mathscr{D})}=G O^{+}\left(V_{1}\right) \times \cdots \times G O^{+}\left(V_{k}\right) \cong\left(\Omega^{+}\left(V_{1}\right) \times \cdots \times \Omega^{+}\left(V_{k}\right)\right) .2^{2 k} .
\end{gathered}
$$

Отсюда

$$
\Omega_{(\mathscr{D})} \cong\left(\Omega^{+}\left(V_{1}\right) \times \cdots \times \Omega^{+}\left(V_{k}\right)\right) \cdot 2^{2(k-1)},
$$

причем

$$
N_{\Omega_{(\mathscr{D})}}\left(K_{i}\right) / C_{\Omega_{(\mathscr{D})}}\left(K_{i}\right) \cong N_{\Omega_{(\mathscr{D})}}\left(K_{i}^{*}\right) / C_{\Omega_{(\mathscr{D})}}\left(K_{i}^{*}\right) \cong P G L_{2}(q), \quad 1 \leqslant i \leqslant k
$$


Отсюда следует, что $N_{I}\left(T^{0}\right)=T$, т.е. теорема в этом случае верна.

Пусть теперь $I=G O(V), n \geqslant 5$ и $a=1$ или 3 . Тогда $I_{\mathscr{D}}$ содержится в стабилизатоpe $I_{\left\{V_{0}, W\right\}}$ в $I$ ортогонального разложения $V=V_{0} \oplus W$, который ввиду [6, предложение 4.1.1] равен $G O\left(V_{0}\right) \times G O^{+}(W)$.

Если $a=1$, то по $\left[6\right.$, предложение 4.1.6] в $G O\left(V_{0}\right) \backslash S O\left(V_{0}\right)$ и $G O^{+}(W) \backslash S O^{+}(W)$ существуют такие отражения $r_{0}$ и $r$ соответственно, что

$$
\Omega_{\left\{V_{0}, W\right\}}=\Omega(W)\left\langle r_{0} r\right\rangle \cong \Omega_{4 k}^{+}(q) .2 .
$$

При $k=1$ ввиду [6, предложение 2.9.1] получаем исключительный случай (е) теоремы. При $k>1$ по уже рассмотренному случаю $a=0$ получаем, что $N_{I}\left(T^{0}\right)=T$, т.е. теорема в этом случае также верна.

Если $a=3$, то по [6, предложение 4.1.6] соответственно в $G O\left(V_{0}\right) \backslash S O\left(V_{0}\right)$ и $G O^{+}(W) \backslash$ $S O^{+}(W)$ существуют такие отражения $r_{0}$ и $r$, а в $S O\left(V_{0}\right) \backslash \Omega\left(V_{0}\right)$ и $S O^{+}(W) \backslash \Omega^{+}(W)$ существуют такие элементы $s_{0}$ и $s$, что

$$
I_{\left\{V_{0}, W\right\}}=\left(\Omega\left(V_{0}\right) \times \Omega(W)\right)\left\langle s_{0} s, r_{0} r\right\rangle \cong\left(\Omega_{3}(q) \times \Omega_{4 k}^{+}(q)\right) \cdot 2^{2} .
$$

Но ввиду [6, предложение 2.9.1] $S O\left(V_{0}\right)=\Omega\left(V_{0}\right)\left\langle s_{0}\right\rangle \cong P G L_{2}(q)$. Отсюда по уже рассмотренному случаю $a=0$ получаем, что $N_{I}\left(T^{0}\right)=T$, т.е. теорема в этом случае верна.

Пусть, наконец, $I=G O^{\delta}(V), n \geqslant 6$ и $a=2$ или 4 . Тогда опять $I_{D}$ содержится в стабилизаторе $I_{\left\{V_{0}, W\right\}}$ в $I$ ортогонального разложения $V=V_{0} \oplus W$, которьй ввиду [6, предложение 4.1.6] равен $G O^{\delta}\left(V_{0}\right) \times G O^{+}(W)$, причем, соответственно в $G O^{\delta}\left(V_{0}\right) \backslash$ $S O^{\delta}\left(V_{0}\right)$ и $G O^{+}(W) \backslash S O^{+}(W)$, существуют такие отражения $r_{0}$ и $r$, а в $S O^{\delta}\left(V_{0}\right) \backslash$ $\Omega^{\delta}\left(V_{0}\right)$ и $S O^{+}(W) \backslash \Omega^{+}(W)$ существуют такие элементы $s_{0}$ и $s$, что

$$
\Omega_{\left\{V_{0}, W\right\}}=\left(\Omega^{\delta}\left(V_{0}\right) \times \Omega^{+}(W)\right)\left\langle s_{0} s, r_{0} r\right\rangle \cong\left(\Omega_{a}^{\delta}(q) \times \Omega_{4 k}^{+}(q)\right) \cdot 2^{2} .
$$

Но ввиду [6, предложение 2.9.1]

$$
G O^{\delta}\left(V_{0}\right)=\Omega\left(V_{0}\right)\left\langle s_{0}, r_{0}\right\rangle \cong D_{2(q-\delta 1)}
$$

при $a=2, \mathrm{a}$

$$
G O^{\delta}\left(V_{0}\right)=G O^{-}\left(V_{0}\right)=\Omega^{-}\left(V_{0}\right)\left\langle s_{0}, r_{0}\right\rangle \quad \text { и } \quad P G O^{-}\left(V_{0}\right) \cong L_{2}\left(q^{2}\right) .2^{2}
$$

при $a=4$. Так как $q^{2} \equiv 1(\bmod 8)$, то силовская 2-подгрупша в $L_{2}\left(q^{2}\right)$ самонормализуема. Отсюда по уже рассмотренному случаю $a=0$ получаем, что $N_{I}\left(T^{0}\right)=T$, т.е. теорема и в этом случае верна.

Теорема 1 доказана. 
3. Доказательство теоремы 2. Пусть $X=\{1,2, \ldots, n\}, S_{n}=S(X)$ и $A_{n}=A(X)$ - соответствующая знакопеременная группа. Если $H \leqslant S_{n}$, то положим $H^{0}=H \cap A_{n}$. При $Y \subset X$ будем отождествлять $S(Y)$ с поточечным стабилизатором $S(X)_{(X \backslash Y)}$ подмножества $X \backslash Y$ в $S(X)$. В случаях $n \leqslant 5$ теорема, очевидно, справедлива (см., например, [7]). Поэтому будем считать, что $n \geqslant 6$. Пусть $n=4 k+a$ с $0 \leqslant a \leqslant 3$ и $X=\{1,2, \ldots, n\}=X_{1} \cup \cdots \cup X_{k} \cup X_{0}$, где $X_{i}=\{4(i-1)+1,4(i-1)+2,4(i-1)+3$, $4(i-1)+4\}$ для $1 \leqslant i \leqslant k$ и $X_{0}=X \backslash \bigcup_{i=1}^{k} X_{i}$. Положим $\mathscr{D}=\left\{X_{0}, X_{1}, \ldots, X_{k}\right\}$. Хорошо известно, что

$$
S(X)_{\mathscr{D}}=S\left(X_{0}\right) \times\left(\left(S\left(X_{1}\right) \times \cdots \times S\left(X_{k}\right)\right): S_{k}\right) \cong S_{a} \times\left(S_{4}<S_{k}\right),
$$

причем $S(X)^{\mathscr{D}}=A(X)^{\mathscr{D}} \cong S_{k}, S\left(X_{i}\right) A(X)=S(X)$ для $1 \leqslant i \leqslant k$ и

$$
S(X)_{(\mathscr{D})}=S\left(X_{0}\right) \times S\left(X_{1}\right) \times \cdots \times S\left(X_{k}\right) \cong S\left(X_{0}\right) \times\left(\left(A\left(X_{1}\right) \times \cdots \times A\left(X_{k}\right)\right) .2^{k}\right) .
$$

Очевидно, что $S(X)_{\mathscr{D}}$ содержит некоторую силовскую 2 -подгруппу $T$ из $S(X)$. Ясно, что $T^{0}$ - силовская 2-подгруппа из $A(X)$, содержащая силовские 2-подгрупшы из $A\left(X_{i}\right)$ для $1 \leqslant i \leqslant k$. Положим $K_{i}=A\left(X_{i}\right)$ для $1 \leqslant i \leqslant k$ и $K=K_{1}$. По аналогии с соответствующим понятием М. Ашбахера назовем класс сопряженных с $K$ подгрупп в $A(X)$ множеством фундаментальны подгрупп в $A(X)$. Положим

$$
\Delta=\operatorname{Fun}_{A(X)}\left(T^{0}\right)=\left\{K^{x} \mid x \in A(X), K^{x} \cap T^{0} \in \operatorname{Syl}_{2}\left(K^{x}\right)\right\} .
$$

Из предыдущего ясно, что $\left\{K_{i} \mid 1 \leqslant i \leqslant k\right\} \subseteq \Delta$. Предположим, что существует элемент $g \in A(X)$ такой, что $K^{g} \in \Delta$ и $K^{g} \neq K_{i}$ для всех $i$. Тогда $K^{g}=A\left(g\left(X_{1}\right)\right)$ и $g\left(X_{1}\right) \neq X_{i}$ для всех $i$. Кроме того, по определению множества $\Delta$ имеем включение

$$
\left\langle\mathrm{O}_{2}\left(K_{i}\right), \mathrm{O}_{2}\left(K^{g}\right)\right\rangle \leqslant T^{0}
$$

для всех $i$. Предположим, что $X_{i} \cap g\left(X_{1}\right) \neq \varnothing$ для некоторого $i$, и покажем существование таких инволюций $a \in O_{2}\left(K_{i}\right)$ и $b \in O_{2}\left(K^{g}\right)$, что порядок элемента $a b$ делится на 3 в противоречие с предыдушим включением. Без ограничения общности можно считать, что $i=1$. Положим $a=(12)(34)$ и подберем подходящую инволюцию $b$.

Пусть сначала $\left|X_{i} \cap g\left(X_{1}\right)\right|=1$. Тогда можно считать, что $X_{i} \cap g\left(X_{1}\right)=\{1\}$ и $g\left(X_{1}\right)=\{1, i, j, k\}$, где $i, j, k \in X \backslash X_{1}$. Положим $b=(1 i)(j k)$. Тогда $a b=(i 21)(34)(j k)$, откуда $|a b|=6$.

Пусть теперь $\left|X_{i} \cap g\left(X_{1}\right)\right|=2$. Тогда можно считать, что $X_{i} \cap g\left(X_{1}\right)=\{1,2\}$ и $g\left(X_{1}\right)=\{1,2, i, j\}$, где $i, j \in X \backslash X_{1}$. Положим $b=(1 i)(2 j)$. Тогда $a b=(1 i 3)(2 j 4)$, откуда $|a b|=3$.

Пусть наконец $\left|X_{i} \cap g\left(X_{1}\right)\right|=3$. Тогда можно считать, что $X_{i} \cap g\left(X_{1}\right)=\{1,2,3\}$ и $g\left(X_{1}\right)=\{1,2,3, i\}$, где $i \in X \backslash X_{1}$. Положим $b=(12)(3 i)$. Тогда $a b=(3 i 4)$, откуда $|a b|=3$.

Мы доказали, что пересечение $g\left(X_{1}\right)$ с $\bigcup_{i=1}^{k} X_{i}$ пустое, т.е. $g\left(X_{1}\right) \subseteq X_{0}$; противоречие с тем, что $\left|X_{1}\right|=4$, a $\left|X_{0}\right| \leqslant 3$. Итак, $\Delta=\left\{K_{i} \mid 1 \leqslant i \leqslant k\right\}$. Из определения множества $\Delta$ непосредственно видно, что $N_{A(X)}\left(T^{0}\right) \leqslant N_{A(X)}(\Delta)$. Так как $C_{X}\left(A\left(X_{i}\right)\right)=X \backslash X_{i}$ и $S\left(A_{i}\right) A(X)=S(X)$ для $1 \leqslant i \leqslant k$, a $X_{0}=\bigcap_{i=1}^{k} C_{X}\left(A\left(X_{i}\right)\right)$, то $N_{S(X)}\left(T^{0}\right) \leqslant N_{S(X)}(\Delta)=S(X)_{\mathscr{D}}$. Рассуждая, как при доказательстве теоремы 1 , получим, что

$$
N_{A(X)_{(\mathscr{D})}}\left(K_{i}\right) / C_{A(X)_{(\mathscr{D})}}\left(K_{i}\right) \cong S_{4}
$$

для $1 \leqslant i \leqslant k$. Отсюда следует, что $N_{S(X)}\left(T^{0}\right)=T$.

Теорема 2 доказана. 
4. Доказательство теоремы 3. Пусть $G$ - одна из конечных простых спорадических групп, $A=\operatorname{Aut}(G), T$ - силовская 2 -подгруппа в $A$ и $T^{0}=T \cap G$. При доказательстве теоремы мы будем ссылаться в основном на [7]. В частности, из [7] видно, что $|A: G| \leqslant 2$.

Для случая $G \cong J_{1}$ утверждение теоремы непосредственно следует из [7].

Пусть $G \cong J_{2}$ или $J_{3}$. Тогда в $G$ есть 2 -скованная максимальная подгруппа $M$ нечетного индекса, изоморфная $2_{-}^{1+4}: A_{5}$, причем $N_{A}(M) \cong 2_{-}^{1+4}: S_{5}$. Поэтому можно считать, что $Z(M)=Z\left(T^{0}\right)$, откуда ввиду теоремы 2 следует утверждение теоремы для этих случаев.

Пусть $G \cong M_{22}, M_{23}$ или $M c L$. Тогда по [11] изоморфньй тип групшы $T^{0}$ единствен, $\left|Z\left(T^{0}\right)\right|=2$ и в $T^{0}$ есть точно две нормальные элементарные подгрупшы $E_{1}$ и $E_{2}$ порядка 16. Ввиду [7] нормализаторы в $G$ подгрупп $E_{1}$ и $E_{2}$ соответственно изоморфны $2^{4}: A_{6}$ и $2^{4}: S_{5}$ при $G \cong M_{22}, 2^{4}: A_{5}$ и $2^{4}:\left(3 \times A_{5}\right): 2$ при $G \cong M_{23}$, а при $G \cong M c L$ в $G$ есть максимальная подгруппа $M$ нечетного индекса, изоморфная $2 \cdot A_{8}$, откуда ввиду теоремы 2 следует утверждение теоремы для этих случаев.

Если $G \cong M_{24}$, то по $[12,(2.2)]$ в $T^{0}$ есть точно две нормальные элементарные подгруппы порядка 64, нормализаторы которых в $G$ ввиду [7] соответственно изоморфны $2^{6}: 3 \cdot S_{6}$ и $2^{6}:\left(L_{3}(2) \times S_{3}\right.$, откуда следует утверждение теоремы для этого случая.

Если $G \cong \mathrm{Co}_{3}$, то по [13, лемма 2.1] $\left|Z\left(T^{0}\right)\right|=2$, а ввиду [7] $N_{G}\left(Z\left(T^{0}\right)\right) \cong 2 \cdot S_{6}(2)$, откуда следует утверждение теоремы для этого случая.

Пусть $G \cong M_{11}, M_{12}, H S, H e, R u, C o_{2}, T h, C o_{1}, J_{4}, F i_{24}^{\prime}, B$ или $M$. Тогда ввиду [7] в $G$ есть 2-скованная максимальная подгруппа $M$ нечетного индекса, изоморфная соответственно $Q_{8}: S_{3} \cong G L_{2}(3), 2_{+}^{1+4} \cdot S_{3},\left(4 \circ Q_{8} \circ Q_{8}\right): S_{5}, 2_{+}^{1+6} \cdot L_{3}(2), 2 \cdot 2^{4+6}: S_{5}$, $2_{+}^{1+8}: S_{6}(2), 2_{+}^{1+8} \cdot A_{9}, 2_{+}^{1+12} \cdot O_{8}^{+}(2), 2_{+}^{1+12} \cdot 3 \cdot M_{22}: 2,2_{+}^{1+12} \cdot 3 U_{4}(3) \cdot 2_{2}, 2_{+}^{1+22} \cdot$ Со $_{2}$ или $2_{+}^{1+22} \cdot C o_{1}$. Поэтому можно считать, что $Z(M)=Z\left(T^{0}\right)$, откуда ввиду теорем 1 и 2 и предыдущего следует утверждение теоремы для этих случаев.

Пусть $G \cong S u z$. Тогда ввиду [7] $|A: G|=2$ и в $G$ есть 2-скованная максимальная подгруппа $M$ нечетного индекса, изоморфная $2_{-}^{1+6} \cdot P S p_{4}(3)$, причем $N_{A}(M) \cong 2_{-}^{1+6}$. $S_{5}(3)$. Поэтому можно считать, что $Z(M)=Z\left(T^{0}\right)$, откуда ввиду теоремы 1 следует утверждение теоремы для этого случая.

Пусть $G \cong H N$. Тогда ввиду [7] $|A: G|=2$ и в $G$ есть 2-скованная максимальная подгруппа $M$ нечетного индекса, изоморфная $2_{+}^{1+8} \cdot\left(A_{5} / 2\right)$, причем $N_{A}(M) \cong 2_{+}^{1+8}$. $\left(A_{5} \times A_{5}\right) \cdot 2^{2}$. Поэтому можно считать, что $Z(M)=Z\left(T^{0}\right)$ и $N_{A}\left(T^{0}\right) \leqslant N_{A}(M)$. По [8, (23.10)] $\operatorname{Aut}\left(2_{+}^{1+8}\right) \cong G O_{8}^{+}(2)$, а по [7] в $G O_{8}^{+}(2)$ имеется единственный класс сопряженных максимальных подгрупш, содержащих подгруппу вида $\left(A_{5} \times A_{5}\right) .2^{2}$, а именно, подгрупп, изоморфных $S_{5} 22$. Теперь легко проверить, что

$$
N_{A}(M) / O_{2}\left(N_{A}(M)\right) \cong P G O_{4}^{+}(3),
$$

откуда ввиду теоремы 1 следует утверждение теоремы для этого случая.

Если $G \cong F i_{22}$, то по [7] максимальные подгрупшы нечетного индекса в $G$ изоморфны $2^{10} \cdot M_{22}, 2^{5+8}:\left(S_{3} \times A_{6}\right)$ или $\left(2 \times 2_{+}^{1+8}\right): S O_{5}(3)$, откуда ввиду теорем 1 и 2 и предыдущего следует утверждение теоремы для этого случая.

Пусть $G \cong F i_{23}$. Тогда по [7] максимальные подгруппы нечетного индекса в $G$ изоморфны $2 \cdot F i_{22}, 2^{2} \cdot U_{6}(2) .2,2^{11} . M_{22}, 2^{6+8}:\left(S_{3} \times A_{7}\right)$ или $\left(2^{2} \times 2_{+}^{1+8}\right) .3 . S O_{5}(3)$, откуда ввиду теорем 1 и предыдущего можно считать, что $N_{G}\left(T^{0}\right)<2^{2} \cdot U_{6}(2) .2$. Но 
по [7] в группе $U_{6}(2) .2$ есть максимальная подгруппа нечетного индекса, изоморфная $2_{+}^{1+8} . S O_{5}(3)$, откуда ввиду теоремы 1 следует утверждение теоремы для этого случая. Теорема 3 доказана.

\section{СПИСОК ЦИТИРОВАННОЙ ЛИТЕРАТУРЫ}

[1] Carter R., Fong P. The Sylow 2-subgroups of the finite classical groups // J. Algebra. 1964. V. 1. № 1. P. 139-151.

[2] Кондратьев А.С., Мазуров В.Д. 2-сигнализаторы конечных простьх групп // Алгебра и логика. 2003. Т. 42. № 5. С. 594-623.

[3] Aschbacher M. On finite groups of Lie type and odd characteristic // J. Algebra. 1980. V. 66. № 2. P. 400-424.

[4] Aschbacher M. A characterization of Chevalley groups over fields of odd order // Ann. Math. 1977. V. 106. № 2-3. P. 353-468; Correction // Ann. Math. 1980. V. 111. № 3. P. 411-414.

[5] Aschbacher M. On the maximal subgroups of the finite classical groups // Invent. Math. 1984. V. 76. P. 469-514.

[6] Kleidman P. B., Liebeck M. W. The Subgroup Structure of Finite Classical Groups. Cambridge: Cambridge University Press, 1990.

[7] Conway J.H., Curtis R.T., Norton S.P., Parker R.A., Wilson R. A. Atlas of Finite Groups. Oxford: Clarendon Press, 1985.

[8] Aschbacher M. Finite Group Theory. Cambridge: Cambridge University Press, 1986.

[9] Carter R. Simple Groups of Lie Type. London: Wiley, 1972.

[10] Dickson L. E. Linear Groups with an Exposition of the Galois Field Theory. New York: Dover, 1958.

[11] Gorenstein D., Harada K. On finite groups with Sylow 2-subgroups of type $\widehat{A}_{n}, n=8,9$, 10 and 11 // J. Algebra. 1971. V. 19. № 2. P. 185-227.

[12] Held D. The simple groups related to $M_{24}$ // J. Algebra. 1969. V. 13. №1. P. 253-296.

[13] Solomon R. Finite groups with Sylow 2-subgroups of type 3 // J. Algebra. 1974. V. 28. № 1. P. 182-198.

Институт математики и механики УрО РАН 